\title{
THEORETICAL \& EXPERIMENTAL INVESTIGATION OF A PROBE-FED RECTANGULAR DR ANTENNA
}

\author{
Zeipang $\mathrm{Wu}^{*}$ and M. H. Neshati \\ Microwave Engineering Group \\ Department of Electrical Engineering \& Electronics \\ University of Manchester Institute of Science \& Technology (UMIST) \\ PO Box 88, Manchester M60 1QD, UK.
}

\section{Introduction:}

Dielectric Resonators (DRs) of high dielectric constant and low loss material are widely used in miniature shielded microwave circuits such as filters and oscillators. In the resent years, open DRs have been proposed as an antenna [1-3], offering a number of advantages over other types, including small size, large bandwidth, simple coupling structure and high radiation efficiency. Compared with other shapes, rectangular resonators is more attractive due to ease of fabrication and have two independent aspect ratios, which could offer flexibility for required radiation characteristics. In this paper a RDRA is studied theoretically and experimentally. The CDWM and the FEM are used to predict the resonance frequency, radiation patterns, directivity, $Q$-factor and impedance bandwidth of the RDRA. The theoretical results are compared with those obtained experimentally.

\section{The Conventional Dielectric Wave-guide Model:}

Figure 1 shows the antenna under investigation. The DR has dielectric constant of $\varepsilon_{r}$ and its dimensions are $a, b$ and $h$ along $x, y$ and $z$-direction respectively excited by a small probe. For theoretical formulation an infinite ground plane is assumed and the effect of the probe is neglected. Image theory is then applied to replace the ground plane with an image of the DR, extending to $z=-h$. The mode of interest is the fundamental $T E_{111}^{y}$ mode [4-5] for the coordinate system shown in Figure 1. In the CDWM, it is assumed that the resonator is truncated of a rectangular dielectric wave-guide [4] having the same transverse dimensions as 
those of the DR, but fields inside the DR are a standing wave type. Boundary conditions are four perfect magnetic wall surfaces at $x= \pm a / 2$ and $z= \pm h$, while on the two others at $y= \pm b / 2$ the tangential fields are continuous leads to the expression of the field components inside the resonator [5]. The variation of fields along $y$-direction is a fraction of a half cycle shown by $\delta$ and it can be shown that wave numbers are

$$
k_{x}=(\pi / a), k_{y}=\delta(\pi / b), k_{z}=(\pi / 2 h)
$$

and the resonance frequency is the solution of the:

$$
\left(\delta \frac{\pi}{b}\right) \tan \left(\delta \frac{\pi}{2}\right)=\sqrt{k_{x}^{2}+k_{z}^{2}-k_{0}^{2}}, \quad k_{x}^{2}+k_{y}^{2}+k_{z}^{2}=k_{0}^{2} \varepsilon_{r}
$$

where $k_{0}$ is free space wave number and $\varepsilon_{r}$ is the relative dielectric constant of the DR. The far-field radiation patterns due to the equivalent magnetic currents on the surfaces of the resonator can be obtained related to the fields inside the resonator. For a resonator with the dimensions of $a=19, b=19$ and $h=9.5 \mathrm{~mm}$ and dielectric constant of 38 , far field co-polarized radiation patterns are shown in Figure 2 and the directivity of the antenna at $\theta=0^{\circ}$ can be further obtained.

\section{The Finite Element Method:}

Using the HP85180A High Frequency Structure Simulator (HFSS), which is a software package for calculating $S$-parameters of passive structures, a full three-dimensional electromagnetic field inside the structure can be calculated. Based on the Finite Element Method (FEM), the HFSS automatically divides the geometric model into a large number of small regions, called element. Each element is a pyramid formed by four equilateral triangles. The HFSS then computes the generalised $S$-matrix from the amount of reflection and transmission at the input port, which is supported by a transmission line having the same cross section as the port defined in the structure. Using an absorber boundary around the structure, the radiated far field patterns and then directivity of the antenna can be obtained.

\section{Simulated and Measured Results:}

For the DR with mentioned dimensions supported by a $100 \mathrm{~mm}$ diameter circular ground plane fed with a probe of $9.5 \mathrm{~mm}$ the simulated and measured co-polarized radiation patterns are shown in Figure 3. Using calibrated dipoles, the antenna gain was measured and the unloaded $Q$-factor of the antenna was calculated from the return loss versus frequency plot using one-port measurement technique [6]. The radiation efficiency was measured using the 
Wheeler Cap method [7]. All other results are summarized in Table 1. It can be seen that the simulation can produce accurate results of radiation patterns of a DRA. But the effect of the finite size ground plane can also be seen especially at the angles near the ground plane.

\section{Conclusion}

Two different modeling techniques are presented for the analysis of RDRAs. Results indicate that CDWM can be used as the first order estimation of the antenna characteristics, but more accurate results can be produced by the HFSS. Also the effect of the size and the geometry of the ground plane needs to taken into consideration, which require further investigation.

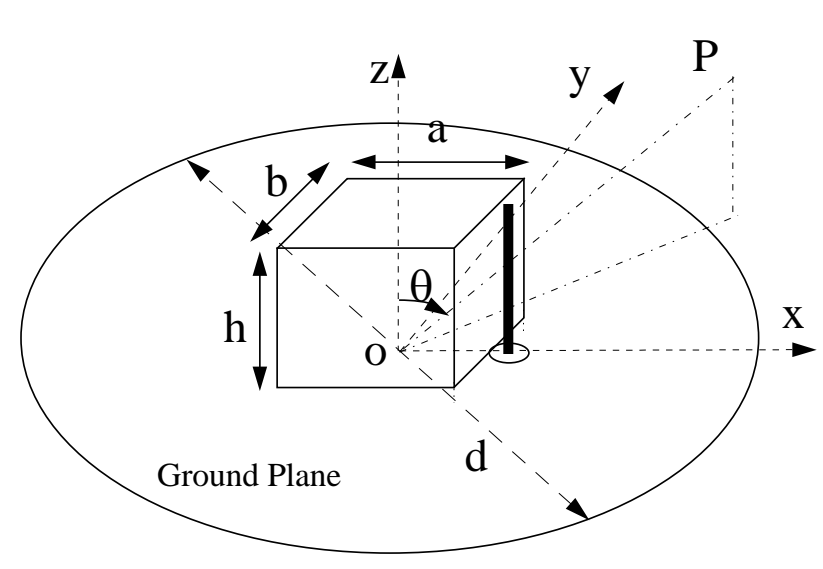

Figure 1. Probe-Fed RDRA

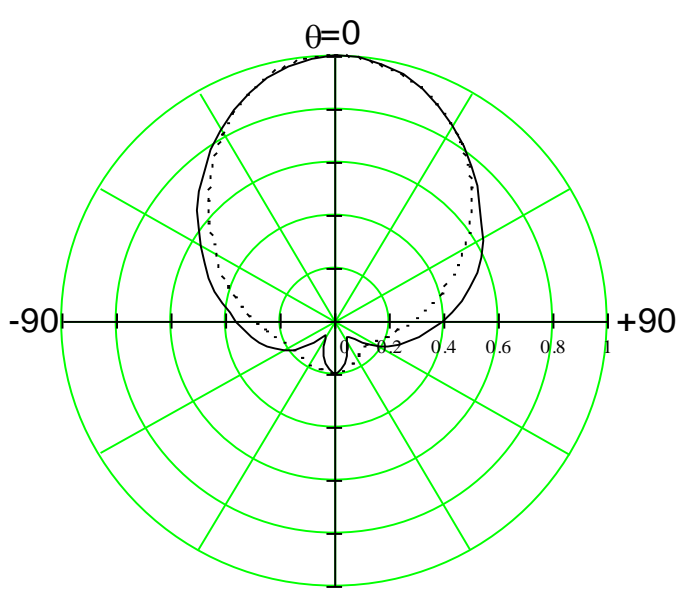

(a)
Figure 2. Theoretical co-polarized Radiation patterns E-Plane

H-Plane -....

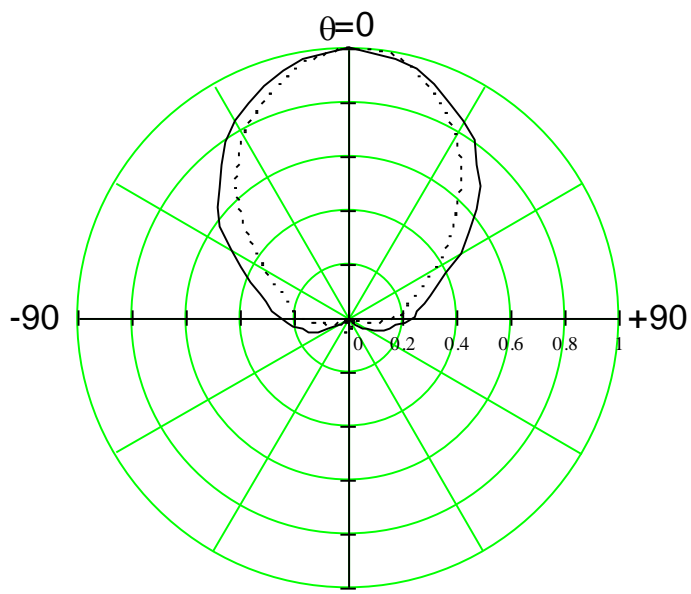

(b)

Figure 3. Co-polarized radiation patterns of the RDRA: a) Simulation b)Measurement

$$
\text { E-Plane }- \text {, H-Plane }----
$$


Table 1: Radiation Parameters of the probe-fed RDRA

\begin{tabular}{|lcccc|}
\hline \multicolumn{2}{|c}{ Parameter } & Theory & Simulation & Measured \\
\hline $\mathbf{S}_{\mathbf{1 1}}(\mathrm{dB})$ & - & -23.247 & -20.585 \\
\hline $\mathbf{f}_{\mathbf{0}}(\mathrm{GHz})$ & 2.022 & 2.078 & 2.206 \\
\hline Q-Factor & 33.088 & 36.793 & 30.906 \\
\hline $\begin{array}{l}\text { BW } \\
\text { (VSWR <2.5) }\end{array}$ & 2.85 & 2.578 & 3.075 \\
\hline Directivity & 2.881 & 4.629 & - \\
\hline Gain & - & - & 3.344 \\
\hline Efficiency (\%) & $\cong 100 \%$ & - & $95 \%$ \\
\hline
\end{tabular}

\section{REFERENCES:}

1. McAllister M. W., Long S. A. And Conway G. L., "Rectangular Dielectric Resonator Antenna", Electronics Letters, Vol. 19, 1983, pp. 218-219.

2. Drossos G., Wu Z., and Davis L.E.,"Cylindrical Dielectric Resonator Antennas: Theoretical Modeling and Experiments", Microwave \& Communication Technologies Conference (M\&RF’97), Wembley Conference Center, 31 September - 1 October 1997, London, UK, pp. 34-39.

3. Mongia R. K., Bhartia P., "Dielectric Resonator Antenna - A Review and General Design Relations to Resonant Frequency and Bandwidth", International Journal of Microwave and Millimetre-Wave Computer Aided Engineering, Vol. 4,1994,pp. 230-247.

4. Mongia R. K., "Theoretical and Experimental Resonance Frequencies of Rectangular Dielectric Resonators", IEE Proce.-H Vol. 139, 1992, pp. 98-104.

5. Mongia R. K., Ittipiboon A., "Theoretical and Experimental Investigations on Rectangular Dielectric Resonator Antennas", IEEE Transactions on Antenna and Propagation, Vol. AP-45, 1997, pp. 1348-1356.

6. Wu Z., Davis L.E. "Automation-orientad Technique for Quality-factor Measure ment of High- $T_{c}$ Superconducting Resonators", IEE Proc.-Sci. Meas. Technol., Vol.141, No. 6, 1994, pp 527-530.

7. Wheeler H. A., "The Radiansphere Around a Small Antenna", Proc. of the IRE 1959, pp. 1325-1331. 